Article

\title{
The Impact of Korean Medicine Treatment on the Incidence of Parkinson's Disease in Patients with Inflammatory Bowel Disease: A Nationwide Population-Based Cohort Study in South Korea
}

\author{
Hyeonseok Noh ${ }^{1}$, Jeongju Jang ${ }^{2}$, Seungwon Kwon ${ }^{1,3}{ }^{\mathbb{C}}$, Seung-Yeon Cho ${ }^{1,3}$, Woo-Sang Jung 1,3, \\ Sang-Kwan Moon ${ }^{1,3}$, Jung-Mi Park ${ }^{1,3}$, Chang-Nam Ko ${ }^{1,3}$, Ho Kim ${ }^{4, *}$ (i) \\ and Seong-Uk Park $1,3, *$ (i) \\ 1 Department of Clinical Korean Medicine, Graduate School, Kyung Hee University, 26, Kyungheedae-ro, \\ Dongdaemun-gu, Seoul 02447, Korea; integritynoh@hanmail.net (H.N.); kkokkottung@hanmail.net (S.K.); \\ sy.cho@khu.ac.kr (S.-Y.C.); wsjung@khu.ac.kr (W.-S.J.); skmoon@khu.ac.kr (S.-K.M.); \\ pajama@khu.ac.kr (J.-M.P.); kcn202@khu.ac.kr (C.-N.K.) \\ 2 Graduate School of Public Health, Seoul National University, 1, Gwanak-ro, Gwanak-gu, Seoul 151-742, \\ Korea; zzooo@snu.ac.kr \\ 3 Department of Cardiology and Neurology, College of Korean Medicine, Kyung Hee University, 26, \\ Kyungheedae-ro, Dongdaemun-gu, Seoul 02447, Korea \\ 4 Department of Public Health Science, Graduate School of Public Health \& Institute of Health and \\ Environment, Seoul National University, 1, Gwanak-ro, Gwanak-gu, Seoul 08826, Korea \\ * Correspondence: hokim@snu.ac.kr (H.K.); seonguk.kr@gmail.com (S.-U.P.)
}

Received: 4 June 2020; Accepted: 25 July 2020; Published: 28 July 2020

\begin{abstract}
We aimed to investigate the association between Korean medicine (KM) treatment and the risk of Parkinson's Disease (PD) in patients with inflammatory bowel disease (IBD) in South Korea. This study analyzed data from the National Health Insurance Service-Senior cohort in South Korea. The 1816 IBD patients enrolled in the analysis comprised 411 who received only conventional treatment (monotherapy group) and 1405 who received both conventional and KM treatments (integrative therapy group). The risk of PD in patients with IBD was significantly lower in the integrative therapy group than in the monotherapy group after adjusting for confounding variables (adjusted hazard ratio (HR), 0.56; 95\% confidence interval $(\mathrm{CI})=0.34-0.92$ ). In the mild Charlson Comorbidity Index (CCI) group, the risk of PD in patients with IBD in the integrative therapy group was 0.39 times lower (adjusted $\mathrm{HR}, 95 \% \mathrm{CI}=0.20-0.77$ ) than that in the monotherapy group. However, there was no significant difference in the risk of PD in patients with IBD between the integrative therapy and monotherapy groups among individuals with severe CCI (adjusted HR, $0.90 ; 95 \% \mathrm{CI}=0.41-1.96)$. IBD patients are at a decreased risk of PD when they receive integrative therapy. KM treatment may prevent PD in IBD patients.
\end{abstract}

Keywords: Parkinson's disease; inflammatory bowel disease; Korean medicine; National Health Insurance Service-Senior cohort; nationwide population-based study

\section{Introduction}

Parkinson's disease (PD), the most common movement disorder, is neuropathologically characterized by progressive loss of dopaminergic neurons and the presence of $\alpha$-synuclein-containing Lewy bodies in the substantia nigra [1]. An estimated 1\% of the population older than 60 years of age is affected by PD. The disease typically causes motor symptoms such as rigidity, resting tremor, bradykinesia, and postural instability, as well as non-motor features including constipation, depression, 
sleep disorders, and autonomic dysfunction. Interactions between environmental and genetic factors, including traumatic brain injury, pesticides, oxidative stress, and genetic mutations contribute to the risk of PD [2].

Inflammatory bowel disease (IBD), comprising Crohn's disease (CD) and ulcerative colitis (UC), is a chronic and recurrent inflammatory disorder. Environmental and microbial factors may interact with genetic components in the pathogenesis of IBD [3]. Communication between the enteric and central nervous system (CNS) activity through the gut-brain axis is associated with immune activity modulation. Chronic intestinal inflammation may contribute to dopaminergic neurodegeneration by disruption of the blood-brain barrier (BBB) [4]. Gastrointestinal (GI) inflammation can trigger $\alpha$-synuclein expression and accumulation in the gut, the aggregates of which subsequently propagate along the vagus nerve to the CNS. The dopaminergic neurodegeneration by 6-hydroxydopamine (6-OHDA) in the CNS may also induce GI tract inflammation by impairing the dorsal motor nucleus of the vagus nerve $[5,6]$. Several cytokines, including interleukin (IL)- $1 \beta$ and tumor necrosis factor (TNF)- $\alpha$, have been associated with PD and IBD [7]. PD and IBD also share key genetic factors including caspase recruitment domain-containing protein 15/nucleotide-binding oligomerization domain-containing protein (CARD15/NOD2) gene polymorphisms as well as LRRK2 mutations [6,7].

Several population-based studies examining the clinical co-occurrence of $\mathrm{PD}$ and IBD have reported an increased risk of PD in patients with IBD [8-12]. However, previous studies had limitations and conflicting results regarding the incidence of PD due to IBD in terms of medication use, comorbidities, and personal health behaviors. To our knowledge, no studies have compared the incidence of PD in IBD patients who received healthcare services other than conventional treatment. Besides conventional medication therapy, Korean medicine (KM) treatment, such as acupuncture, moxibustion, cupping, psychotherapy, and insured herbal preparations has been widely used for the treatment of IBD in South Korea. Therefore, this nationwide population-based study aimed to investigate the association between KM treatment and the risk of PD in patients with IBD in South Korea and provide evidence for the preventive advantage of KM treatment on the incidence of PD in patients with IBD.

\section{Methods}

\subsection{Data Sources}

This retrospective population-based cohort study was based on data from the nationwide administrative claims-based database of the National Health Insurance Service (NHIS) in South Korea. The NHIS database is categorized into insured employees, insured self-employed individuals, or medical aid beneficiaries and includes information on personal demographics, inpatient and outpatient medical services, prescription records, and procedures and diagnosis according to International Classification of Diseases, 10th revision (ICD-10) codes [13]. Health-related information obtained using questionnaires (physical activity, alcohol consumption, cigarette smoking, coffee drinking, and self-reporting of past medical history and family history), anthropometry, blood pressure measurements, and clinical laboratory results were also included in the general health screening database. Since the data were de-identified, subject consent was not required.

The NHIS-Senior cohort statistically represents the population aged more than 60 years selected by $10 \%$ simple random sampling from a total of approximately 5.5 million South Korean subjects aged 60 years and over in 2002. The cohort was followed until 2015 unless death or disqualification for National Health Insurance, such as emigration [14]. The study protocol was exempted from the Institutional Review Board (IRB) of Kyung Hee University Korean Medicine Hospital at Gangdong (IRB No. 2019-09-009).

\subsection{Study Population}

Patients diagnosed with IBD were identified based on ICD-10 codes (K50 for CD and K51 for UC) as the primary or secondary diagnosis between 1 January 2002, and 31 December 2006, from the 
NHIS-Senior cohort database according to two or more outpatient clinic visits or hospitalization for more than two days. All IBD patients were followed up from 1 January 2007, to 31 December 2015. Patients with PD following IBD diagnosis were defined according to ICD-10 codes G20 during the follow-up period. The exclusion criteria were primary or secondary diagnoses of PD prior to IBD or death from 1 January 2002, to 31 December 2006.

\subsection{Definitions of Variables}

The baseline characteristics investigated in this study included sex, age, comorbidities, Charlson Comorbidity Index (CCI), medication use, hospitalization (days), outpatient use (days), and number of hospitals visited. As described previously, the comorbidities considered in this study included alcohol-related diseases (ICD-10 codes F10 and K70), cardiovascular diseases (ICD-10 codes I21-22, I252, I099, I110, I130, I132, I255, I420, I425-I429, I43, I50, G45-46, I60-I69, H340), chronic kidney diseases (ICD-10 codes N032-N037, N052-N057, N18-19, N250, I120, I131, Z490-Z492, Z940, Z992), chronic obstructive pulmonary disease (COPD) (ICD-10 codes J40-J49), dementia (ICD-10 codes F00-F03, G30, G31), depression (ICD-10 codes F32-33, F34.1), diabetes mellitus (ICD-10 codes E10-E14), hyperlipidemia (ICD-10 codes E780-E785), and hypertension (ICD-10 codes I10-I15) [15-19]. According to the $\mathrm{CCI}$, the severity of the comorbidities was stratified as mild ( 0 or 1 point) or severe ( 2 points or more). Information about the drugs used to treat IBD (5-aminosalicylic acid, corticosteroid, immunomodulator, anti-TNF- $\alpha$ agent) is described in Supplementary Table S1 [20,21].

\subsection{Exposures and Outcomes}

The primary exposure examined was the cumulative use of KM treatment during the follow-up period. Each IBD patient's exposure to KM treatment was determined using the National Health Insurance Electronic Data Interchange (EDI) codes provided by the Health Insurance Review \& Assessment Service. The types of KM were classified as acupuncture, moxibustion, cupping, psychotherapy, and insured herbal preparations (Supplementary Table S2). The eligible IBD patients were divided into a monotherapy group that received only conventional treatment and an integrative therapy group that received both conventional and KM treatments. The outcome was newly developed PD (ICD-10 codes G20) as a primary or secondary diagnosis between 1 January 2007, and 31 December 2015, or until withdrawal from the insurance system.

\subsection{Statistical Analysis}

The differences in baseline characteristics between the two groups were compared by the $t-$ and chi-square tests for continuous and categorical variables, respectively. The incidence rates of PD per 1000 person-years were calculated in each group. Cox proportional hazard regression analysis was used to calculate the adjusted hazard ratio (HR) and $95 \%$ confidence interval (CI) to determine whether KM treatment was an independent risk factor for the incidence of PD in patients with IBD after adjusting for sex, age, comorbidities, CCI, medication use, hospitalization (days), outpatient use (days), and number of hospitals visited. The cumulative incidences of PD between two groups were analyzed using the Kaplan-Meier method, and log-rank tests were used to compare differences in the curves. $p$-values $<0.05$ were considered statistically significant. Statistical analysis was performed using SAS for Windows (version 9.4, SAS Institute, Inc., Cary, NC, USA) and R version 3.5.2 for Windows (R Core Team, Vienna, Austria, 2018).

\section{Results}

\subsection{Study Description}

A total of 558,147 individuals over 60 years of age were identified in the NHIS-Senior cohort database from 1 January 2002, to 31 December 2006. Among them, 1995 were diagnosed with IBD based on ICD-10 codes (K50 for CD and K51 for UC) as primary or secondary diagnosis during the study 
period. After excluding 23 patients with pre-existing PD and 156 patients who died during the baseline period, the remaining 1816 eligible patients included 411 who received only conventional treatment (monotherapy group) and 1405 who received both conventional and KM treatments (integrative therapy group). The inclusion and exclusion criteria of the study population are shown in Figure 1.

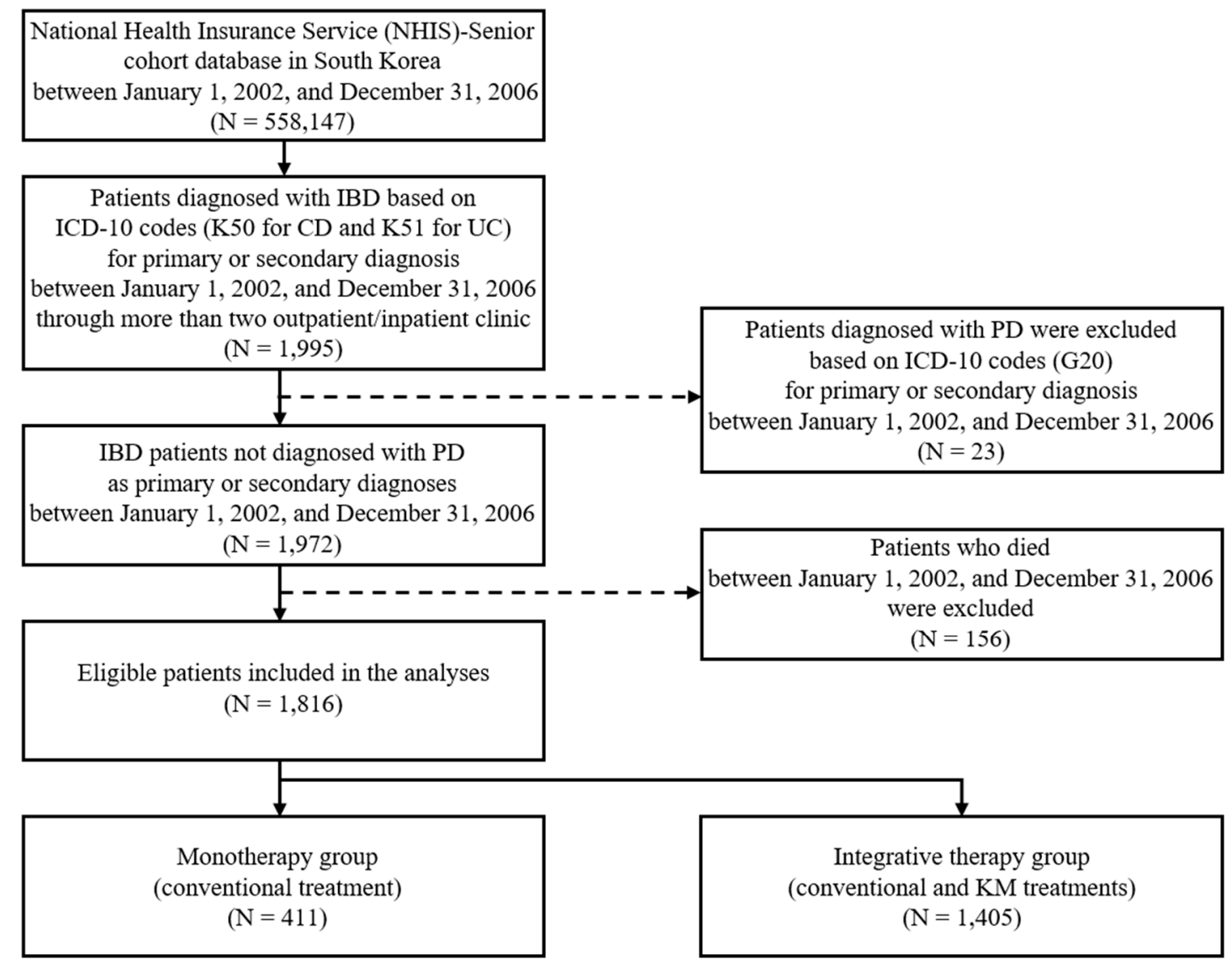

Figure 1. Flowchart of the study population. CD: Crohn's disease, IBD: Inflammatory bowel disease, ICD-10: International Classification of Diseases, $10^{\text {th }}$ revision, KM: Korean medicine, PD: Parkinson's disease, UC: Ulcerative colitis.

\subsection{Baseline Characteristics of the Study Population}

The demographics of the study population at baseline are presented in Table 1 . There were significant differences between the two groups in sex, COPD, CCI, and medication use ( $p$-value $<0.001$ ). The distributions of the other variables did not differ significantly between the two groups. 
Table 1. Baseline study population demographics.

\begin{tabular}{|c|c|c|c|}
\hline & $\begin{array}{l}\text { Monotherapy Group } \\
\qquad(n=411)\end{array}$ & $\begin{array}{l}\text { Integrative Therapy } \\
\text { Group }(n=1405)\end{array}$ & $p$-Value \\
\hline Sex & & & $<0.001$ \\
\hline Male & $242(58.9 \%)$ & $526(37.4 \%)$ & \\
\hline Female & $169(41.1 \%)$ & $879(62.6 \%)$ & \\
\hline Age & & & 0.061 \\
\hline $60-64$ & $167(40.6 \%)$ & $578(41.1 \%)$ & \\
\hline $65-69$ & $120(29.2 \%)$ & $419(29.8 \%)$ & \\
\hline $70-74$ & $63(15.3 \%)$ & $254(18.1 \%)$ & \\
\hline $75-79$ & $41(36.7 \%)$ & $121(8.6 \%)$ & \\
\hline$\geq 80$ & $20(4.9 \%)$ & $33(2.4 \%)$ & \\
\hline \multicolumn{4}{|l|}{ Comorbidity } \\
\hline Alcohol-related diseases & $20(4.9 \%)$ & $75(5.3 \%)$ & 0.706 \\
\hline Cardiovascular diseases & $137(33.3 \%)$ & $580(41.3 \%)$ & 0.004 \\
\hline Chronic kidney diseases & $44(10.7 \%)$ & $163(11.6 \%)$ & 0.615 \\
\hline $\begin{array}{l}\text { Chronic obstructive } \\
\text { pulmonary diseases }\end{array}$ & $219(53.3 \%)$ & $881(62.7 \%)$ & $<0.001$ \\
\hline Dementia & $19(4.6 \%)$ & $74(5.3 \%)$ & 0.602 \\
\hline Depression & $44(10.7 \%)$ & $230(16.4 \%)$ & 0.005 \\
\hline Diabetes mellitus & $125(30.4 \%)$ & $467(33.2 \%)$ & 0.283 \\
\hline Hyperlipidemial & $90(21.9 \%)$ & $364(25.9 \%)$ & 0.099 \\
\hline Hypertension & $242(58.9 \%)$ & $853(60.7 \%)$ & 0.505 \\
\hline $\begin{array}{l}\text { Charlson Comorbidity } \\
\text { Index }\end{array}$ & & & $<0.001$ \\
\hline 0 & $118(28.7 \%)$ & $246(17.5 \%)$ & \\
\hline 1 & $128(31.1 \%)$ & $440(31.3 \%)$ & \\
\hline 2 & $83(20.2 \%)$ & $378(26.9 \%)$ & \\
\hline$\geq 3$ & $82(20.0 \%)$ & $341(24.3 \%)$ & \\
\hline Biomedicine Drug & & & $<0.001$ \\
\hline Yes & $299(72.8 \%)$ & $1132(80.6 \%)$ & \\
\hline No & $112(27.3 \%)$ & $273(19.4 \%)$ & \\
\hline $\begin{array}{l}\text { Treatment period in } \\
\text { biomedicine (days) }\end{array}$ & & & 0.207 \\
\hline 1 & $204(49.6 \%)$ & $747(53.2 \%)$ & \\
\hline$\geq 2$ & $207(50.4 \%)$ & $658(46.8 \%)$ & \\
\hline \multicolumn{4}{|l|}{$\begin{array}{l}\text { Hospitalization period } \\
\text { in biomedicine (days) }\end{array}$} \\
\hline 0 & $360(87.6 \%)$ & $1281(91.2 \%)$ & 0.03 \\
\hline$\geq 1$ & $51(12.4 \%)$ & $124(8.8 \%)$ & \\
\hline $\begin{array}{c}\text { \# of visiting } \\
\text { biomedicine hospital }\end{array}$ & & & 0.158 \\
\hline 1 & $362(88.1 \%)$ & $1271(90.5 \%)$ & \\
\hline$\geq 2$ & $49(11.9 \%)$ & $134(9.5 \%)$ & \\
\hline
\end{tabular}

\subsection{Incidence and Risk of PD in Patients with IBD According to KM Treatment}

During the follow-up period, $55(3.91 \%)$ and $25(6.08 \%)$ patients in the integrative therapy and monotherapy groups, respectively, developed PD. The Kaplan-Meier survival curves for the risk of PD in patients with IBD differed significantly between the two groups, and the risk of PD in patients with IBD was significantly lower in the integrative therapy group $(\log$-rank $p$-value $=0.031)$ (Figure 2$)$. 


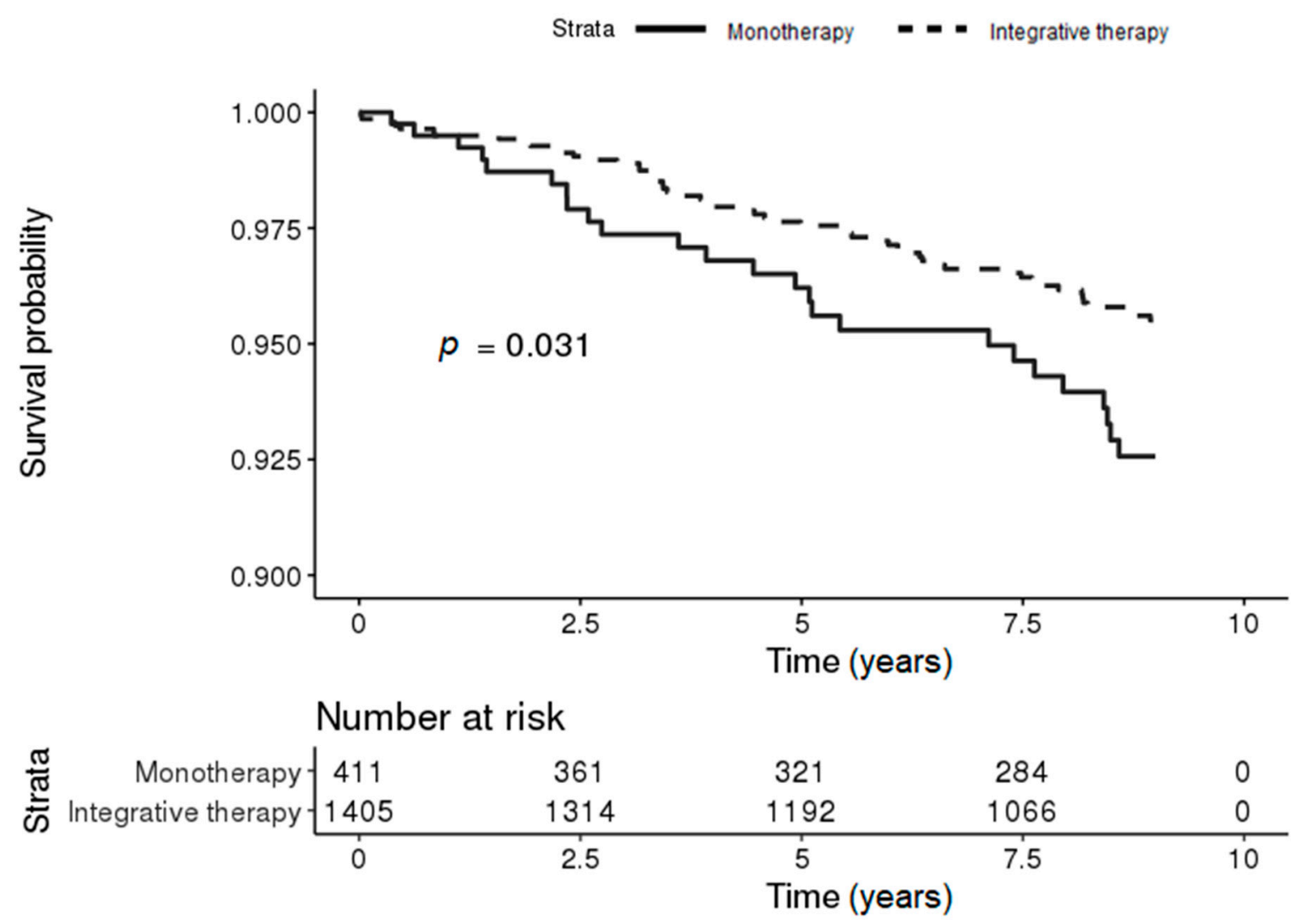

Figure 2. Kaplan-Meier survival curves for the incidence of PD in patients with IBD according to treatment (conventional [monotherapy] vs. integrated [conventional and Korean medicine]).

IBD patients aged 65 years and over in the integrative therapy group were 0.51 times less likely to develop PD compared to the risk in the monotherapy group after adjusting for sex, comorbidities, medication use, hospitalization (days), outpatient use (days), and number of hospitals visited (95\% $\mathrm{CI}=0.28-0.94 ; 6.5$ vs. 12.5 per 1000 person-years). Moreover, IBD patients in the integrative therapy group who used medication had a 0.54 -fold lower risk of PD than those in the monotherapy group after adjusting for sex, age, comorbidities, hospitalization (days), outpatient use (days), and number of hospitals visited (95\% CI $=0.30-0.96 ; 4.7$ vs. 8.3 per 1000 person-years). After adjusting for confounding variables, the adjusted HR for developing PD in patients with IBD was 0.56 times lower in the integrative therapy group than in the monotherapy group $(95 \% \mathrm{CI}=0.34-0.92 ; 5.1$ vs. 8.4 per 1000 person-years) (Table 2).

\subsection{Sensitivity Analysis}

The sensitivity analysis showed that IBD patients in the integrated therapy group treated with KM more than twice had a 0.51-fold lower risk of PD than patients in the monotherapy group after adjusting for confounding variables ( $95 \% \mathrm{CI}=0.31-0.82 ; 4.9$ vs. 8.8 per 1000 person-years). Cox regression analysis also showed that the risks of PD occurrence in IBD patients in the integrated therapy group who received KM more than three and more than five times were 0.57 -fold ( $95 \% \mathrm{CI}=0.35-0.92$; 5 vs. 8 per 1000 person-years) and 0.62 -fold ( $95 \% \mathrm{CI}=0.39-0.98 ; 5$ vs. 7.5 per 1000 person-years) lower, respectively, than those in the monotherapy group after adjusting for confounding variables. No statistically significant difference in the risk of PD in patients with IBD was observed between the integrative therapy and monotherapy groups among IBD patients treated with KM more than 10 times (adjusted HR, $0.68 ; 95 \% \mathrm{CI}=0.43-1.07 ; 5$ vs. 6.7 per 1000 person-years) (Table 3 ). 
Table 2. Risk of PD in patients with IBD according to KM treatment.

\begin{tabular}{|c|c|c|c|c|c|c|c|c|c|c|}
\hline & \multicolumn{4}{|c|}{ Monotherapy } & \multicolumn{4}{|c|}{ Integrative Therapy } & \multirow[b]{2}{*}{$\begin{array}{c}\text { Crude HR } \\
(95 \% \text { CI })\end{array}$} & \multirow[b]{2}{*}{$\begin{array}{c}\text { Adjusted }{ }^{b} \mathrm{HR} \\
(95 \% \mathrm{CI})\end{array}$} \\
\hline & $n$ & Events & Person-Year & Incidence $^{a}$ & $n$ & Events & Person-Year & Incidence & & \\
\hline $\begin{array}{l}\text { All } \\
\text { Sex }\end{array}$ & 411 & 25 & 2968 & 8.4 & 1405 & 55 & 10,890 & 5.1 & $0.60(0.37-0.96)$ & $0.56(0.34-0.92)$ \\
\hline Male & 242 & 13 & 1718 & 7.6 & 526 & 19 & 3980 & 4.8 & $0.63(0.31-1.28)$ & $0.57(0.28-1.17)$ \\
\hline $\begin{array}{l}\text { Female } \\
\text { Age }\end{array}$ & 169 & 12 & 1249 & 9.6 & 879 & 36 & 6910 & 5.2 & $0.54(0.28-1.04)$ & $0.58(0.29-1.14)$ \\
\hline$<65$ & 197 & 8 & 1607 & 5.0 & 664 & 20 & 5489 & 3.6 & $0.73(0.32-1.66)$ & $0.77(0.33-1.82)$ \\
\hline$\geq 65$ & 214 & 17 & 1361 & 12.5 & 741 & 35 & 5392 & 6.5 & $0.52(0.29-0.92)$ & $0.51(0.28-0.94)$ \\
\hline $\begin{array}{l}\text { Medicatior } \\
\text { use }\end{array}$ & & & & & & & & & & \\
\hline 0 & 112 & 7 & 806 & 8.7 & 273 & 14 & 2084 & 6.7 & $0.77(0.31-1.92)$ & $0.79(0.30-2.08)$ \\
\hline 1 & 299 & 18 & 2162 & 8.3 & 1132 & 41 & 8607 & 4.7 & $0.56(0.32-0.97)$ & $0.54(0.30-0.96)$ \\
\hline
\end{tabular}

${ }^{\text {a }}$ Per 1000 person-years; ${ }^{\mathbf{b}}$ Model adjusted for sex, age, alcohol, cardiovascular, chronic kidney, chronic obstructive pulmonary disease, dementia, depression, diabetes mellitus, hyperlipdemial, hypertension, medication use, hospitalization medical use, outpatient medical use, and number of hospitals visited. HR: hazard ratio. 
Table 3. Risk of PD in patients with IBD according to the number of KM treatments.

\begin{tabular}{|c|c|c|c|c|c|c|c|c|c|c|}
\hline & \multicolumn{4}{|c|}{ Monotherapy } & \multicolumn{4}{|c|}{ Integrative Therapy } & \multirow[b]{2}{*}{$\begin{array}{c}\text { Crude HR } \\
\text { (95\% CI) }\end{array}$} & \multirow[b]{2}{*}{$\begin{array}{c}\text { Adjusted }^{\mathrm{b}} \mathrm{HR} \\
(95 \% \mathrm{CI})\end{array}$} \\
\hline & $n$ & Events & Person-Years & Incidence $^{a}$ & $n$ & Events & Person-Years & Incidence & & \\
\hline $\begin{array}{c}\text { Main analysis } \\
\# \text { of } K M^{c} \geq 1\end{array}$ & 411 & 25 & 2968 & 8.4 & 1405 & 55 & 10,890 & 5.1 & $0.60(0.37-0.96)$ & $0.56(0.34-0.92)$ \\
\hline \multicolumn{11}{|c|}{ Sensitivity analysis } \\
\hline$\#$ of $K M \geq 2$ & 437 & 28 & 3170 & 8.8 & 1379 & 52 & 10,688 & 4.9 & $0.55(0.35-0.87)$ & $0.51(0.31-0.82)$ \\
\hline$\#$ of $K M \geq 3$ & 496 & 29 & 3613 & 8 & 1320 & 51 & 10,245 & 5 & $0.62(0.39-0.98)$ & $0.57(0.35-0.92)$ \\
\hline$\#$ of $K M \geq 5$ & 604 & 33 & 4412 & 7.5 & 1212 & 47 & 9447 & 5 & $0.66(0.43-1.04)$ & $0.62(0.39-0.98)$ \\
\hline$\#$ of $K M \geq 10$ & 822 & 41 & 6105 & 6.7 & 994 & 39 & 7754 & 5 & $0.75(0.48-1.16)$ & $0.68(0.43-1.07)$ \\
\hline
\end{tabular}

${ }^{a}$ Per 1000 person-years; ${ }^{\mathbf{b}}$ Model adjusted for sex, age, alcohol, cardiovascular, chronic kidney, chronic obstructive pulmonary disease, dementia, depression, diabetes mellitus, hyperlipdemial, hypertension, medication use, hospitalization medical use, outpatient medical use, and number of hospitals visited; ${ }^{\mathrm{c}}$ Number of Korean medicine treatments. 


\subsection{Subgroup Analyses}

The subgroup analyses divided the eligible IBD patients into two groups according to comorbidity severity based on the CCI (mild: 0 or 1 points, severe: 2 points or more).

3.5.1. Comparison of the Incidence of PD in Patients with IBD According to KM Treatment in the Mild CCI Group

In the mild CCI group, $20(2.92 \%)$ and $17(6.91 \%)$ IBD patients in the integrative therapy and monotherapy groups, respectively, developed PD during the follow-up period. The Kaplan-Meier survival curves for the risk of PD in patients with IBD differed significantly between the two groups, and the risk of PD in patients with IBD was significantly lower in the integrative therapy group $(\log$-rank $p$-value $=0.0045)($ Figure 3$)$.

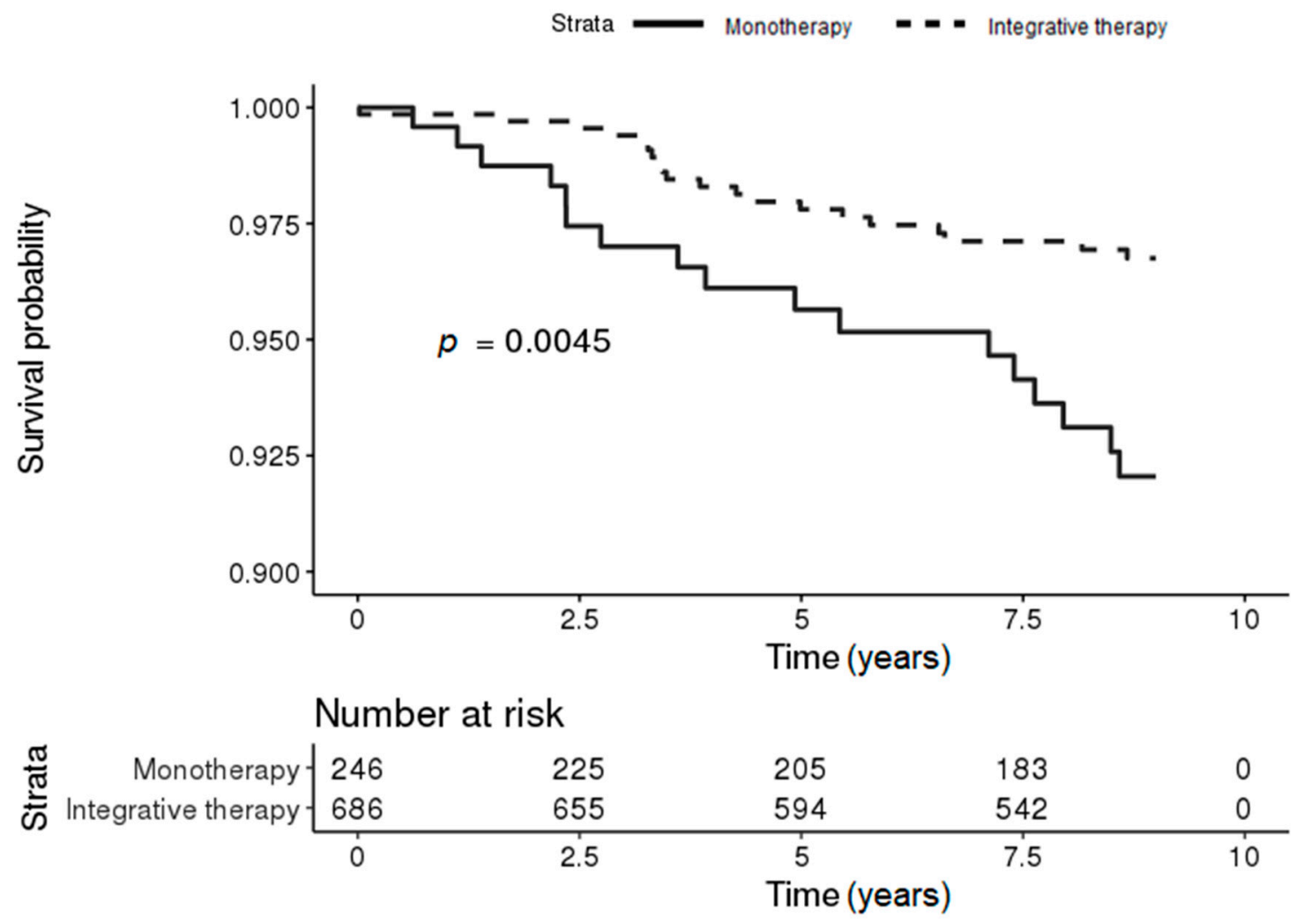

Figure 3. Kaplan-Meier survival curves for the incidence of PD in patients with IBD according to KM treatment in the mild Charlson Comorbidity Index (CCI) group (conventional [monotherapy] vs. integrated [conventional and Korean medicine]).

3.5.2. Comparison of the Incidence of PD in Patients with IBD According to KM Treatment in the Severe CCI Group

In the severe CCI group, $35(4.87 \%)$ and 8 (4.85\%) IBD patients the integrative therapy and monotherapy groups, respectively, developed PD during the follow-up period. The Kaplan-Meier survival curves for the risk of PD in patients with IBD showed no significant difference between the two groups $(\log$-rank $p$-value $=0.73)($ Figure 4$)$. 


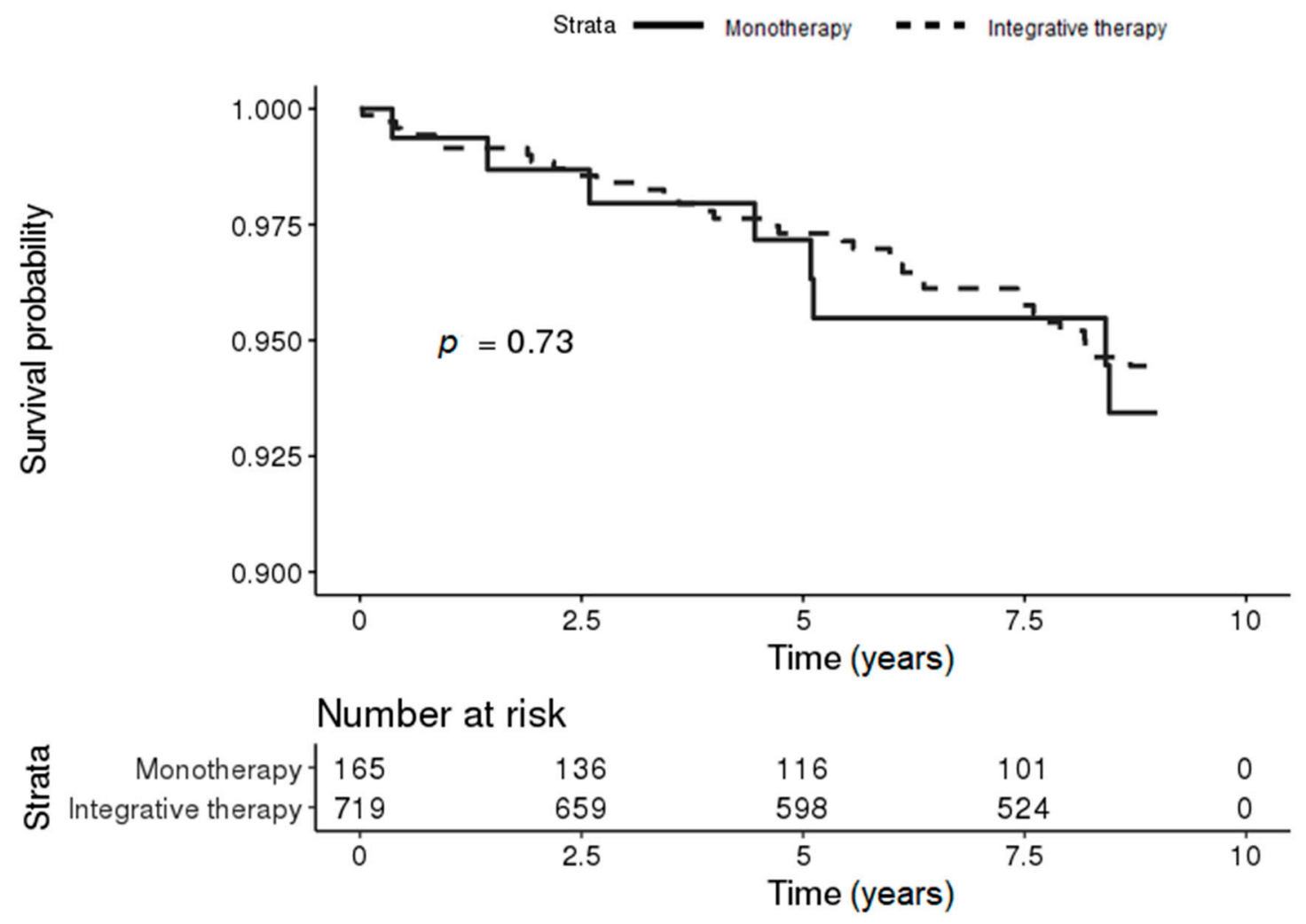

Figure 4. Kaplan-Meier survival curves for the incidence of PD in patients with IBD according to $\mathrm{KM}$ treatment in the severe CCI group (conventional [monotherapy] vs. integrated [conventional and Korean medicine]).

\subsubsection{Risk of PD in Patients with IBD According to KM Treatment in the CCI Group}

In the mild CCI group, Cox regression analysis showed that the risk of PD in patients with IBD in the integrative therapy group was 0.39 times lower than that in the monotherapy group after adjusting for confounding variables $(95 \% \mathrm{CI}=0.20-0.77 ; 3.7$ vs. 9.1 per 1000 person-years). After adjusting for confounding variables, no statistically significant difference in the risk of PD in patients with IBD was observed between the integrative therapy and monotherapy groups among individuals with severe CCI (adjusted HR, 0.90; 95\% CI = 0.41-1.96; 6.4 vs. 7.3 per 1000 person-years) (Table 4 ). 
Table 4. Risk of PD in patients with IBD according to KM treatment in the CCI group.

\begin{tabular}{|c|c|c|c|c|c|c|c|c|c|c|c|}
\hline & \multicolumn{4}{|c|}{ Monotherapy } & \multicolumn{4}{|c|}{ Integrative Therapy } & \multirow{2}{*}{$\begin{array}{l}\text { Crude HR } \\
(95 \% \mathrm{CI})\end{array}$} & \multirow{2}{*}{\multicolumn{2}{|c|}{$\begin{array}{c}\text { Model 1 }^{a} \text { Model }^{\text {b }} \\
\text { Adjusted HR (95\% CI) }\end{array}$}} \\
\hline & $n$ & Events & Person-Year & Incidence & $n$ & Events & Person-Year & Incidence & & & \\
\hline \multicolumn{12}{|l|}{ CCI } \\
\hline Low & 246 & 17 & 1872 & 9.1 & 686 & 20 & 5444 & 3.7 & $0.40(0.21-0.77)$ & $0.37(0.19-0.71)$ & $0.39(0.20-0.77)$ \\
\hline High & 165 & 8 & 1096 & 7.3 & 719 & 35 & 5447 & 6.4 & $0.87(0.41-1.89)$ & $0.91(0.42-1.99)$ & $0.90(0.41-1.96)$ \\
\hline
\end{tabular}

a Model 1 adjusted for sex, and age; ${ }^{b}$ Model 2 adjusted for sex, age, medication use, hospitalization medical use, outpatient medical use, and number of hospitals visited.

CI: confidence interval. 


\section{Discussion}

The results of this nationwide population-based cohort study showed that IBD patients treated with integrative therapy were significantly (0.56-fold) less likely to develop PD compared to IBD patients who received only monotherapy, after adjusting for sex, age, comorbidities, medication use, hospitalization (days), outpatient use (days), and number of hospitals visited in the population of South Korea. Moreover, the subgroup analysis showed that IBD patients with mild CCI treated with integrative therapy had a 0.39-fold lower risk of developing PD.

Previous nationwide population-based cohort studies have also investigated the increased risk of PD among IBD patients [8-12]. Lin et al. (2016) showed a 35\% increase in the risk of PD in patients with IBD compared to that in patients without IBD in Taiwan, especially for CD [8]. In a US cohort study (2018), patients with IBD were 28\% more likely to develop PD than were individuals without IBD and patients with IBD exposed to anti-tumor necrosis factor-alpha (TNF- $\alpha$ ) agents had a $78 \%$ decrease in the risk of PD occurrence compared to the risk in unexposed patients [9]. However, neither study considered the number of hospitals visited, which might have resulted in surveillance bias effects on the risk of PD in patients with IBD. A Swedish cohort study (2018) reported a 30\% increase in the risk of PD in patients with UC compared to that in controls, but these effects vanished after adjusting for the number of healthcare visits during the follow-up period to reduce potential surveillance bias [10]. A Danish cohort study (2018) also showed that patients with IBD had a $22 \%$ increase in the risk of PD as compared to the risk in non-IBD individuals [11]. The results have also led to debates over surveillance bias on the risk of PD in patients with IBD not considering the number of healthcare visits. Park et al. (2019) showed an increased risk of PD in patients with IBD in South Korea, which is an association that remained significant after adjusting for healthcare use. Furthermore, IBD patients receiving steroid or anti-TNF agents who may have had severe IBD showed a significantly reduced risk of PD compared to the risk in patients with mild disease [12].

The potential regulatory effect of cytokines on the growth and differentiation of the cells in the nervous system was reported by findings [22-24]. Based on these results, the association between neurons and immune system has continuously been investigated up to now. In the pathogenesis of IBD and PD, the inflammatory process that underlies both diseases shares some pro-inflammatory cytokines such as TNF- $\alpha$ and IL-1 $\beta$. The central nigrostriatal dopaminergic degeneration followed by bowel inflammation is associated with increased TNF- $\alpha$ and IL-1 $\beta$. Intestinal inflammation might be caused by alterations in the gut microbiota that regulate pathways that promote $\alpha$-synuclein aggregation. $\alpha$-Synuclein deposits present in the bowel wall may be disseminated through the vagus nerve and might also cause dopaminergic neurodegeneration in PD patients [25].

Currently, conventional medication therapy is used as the first option in the treatment of chronic and recurrent IBD for improving patients' quality of life. According to a study investigating the medication use patterns in IBD patients based on data from a nationwide administrative claims-based database of the NHIS in South Korea, 5-aminosalicylic acid was most commonly used, followed by corticosteroids, immunomodulators, and anti-TNF- $\alpha$ agent [20]. However, long-term corticosteroid therapy for the treatment of IBD can lead to venous thromboembolism (VTE), infections, and fragility fractures [26]. The use of anti-TNF (infliximab and adalimumab) agents also significantly increases the risk of tuberculosis (TB) infection and malignancies [27,28].

Several studies investigated the efficacy and the mechanisms of KM treatment for the treatment of IBD. Acupuncture and moxibustion treatment showed better overall efficacy than 5-aminosalicylic acid in treating IBD [29]. According to a resting-state functional magnetic resonance imaging (fMRI) study in CD patients, electro-acupuncture and moxibustion mainly modulated the brain homeostatic afferent processing network and default mode network, respectively [30]. These results suggested that electro-acupuncture and moxibustion may help to improve clinical results in CD patients through different central integration patterns. Acupuncture and moxibustion also inhibited levels of IL-17 and retinoid-related orphan receptor gamma (ROR $\gamma t$ ) but induced forkhead box P3 (FOXP3) expression in the intestinal mucosa of patients with CD [31]. In addition, herb-partitioned moxibustion combined 
with acupuncture induces mucosa inflammation remission and repairs the tight junction barrier structure in intestinal epithelial mucosa, increasing the expression of zonula occludens-1 mRNA [32]. Another systematic review showed that complementary and alternative medicine (CAM) treatments integrated with conventional medicine might be safe and beneficial in IBD patients [33].

Acupuncture transmits signals into the vagus nerve and attenuates inflammatory responses, as measured by TNF- $\alpha$ productions in the serum and spleen. The vagus nerve acts as a bridge between the neural and internal organs for homeostatic regulation and immune systems [34]. Moxibustion also relieves visceral hypersensitivity and inhibits intestinal inflammation by regulating the relative abundance of the gut microbiota. Through these underlying mechanisms, acupuncture and moxibustion might be beneficial for IBD treatment and PD prevention [35].

In the present study, there were no significant differences between the integrative therapy and monotherapy groups among individuals with severe CCI. In the sensitivity analysis, Cox regression analysis showed more significant differences between the integrative therapy and monotherapy groups through $K M \geq 2$, but the adjusted HR gradually increased for $K M \geq 3, \geq 5$, and $\geq 10$ treatments. These findings might be explained by surveillance bias based on the following idea: "the more we look, the more we find" [36]. Unhealthy individuals are more concerned about their health so are more likely to use medical services, including KM treatment. Therefore, surveillance bias should be considered when evaluating outcome measures [37].

To our knowledge, this study is the first to compare the incidence of PD in IBD patients who received healthcare services other than conventional treatment. However, this study has several limitations. First, the NHIS-Senior cohort may have selection or ascertainment bias in health screening information due to issues regarding service eligibility. Furthermore, the results rely on the completeness and accuracy of data obtained from the nationwide administrative claims-based database of the NHIS. The disease codes listed in the cohort might not accurately represent the patients' medical status. Second, information on potential confounding variables on personal health behaviors or lifestyle including physical activity, alcohol consumption, smoking, coffee drinking, socioeconomic status, occupation, and family medical history was limited because those data were obtained from self-reporting questionnaires in nationwide health screening. In addition, genetic factors including CARD15/NOD2 and LRRK2 were not evaluated due to data availability. The patterns of personal health behaviors and genetic factors have differed between the groups. Third, because the KM treatment patterns were mixed forms, such as acupuncture, moxibustion, cupping, psychotherapy and insured herbal preparations, the effect of each KM treatment could not be verified. Fourth, the effects of integrative treatment retention and adherence cannot be accurately quantified. Considering these limitations, the results of this study should be interpreted cautiously. Further investigations are needed to determine how KM treatment influences changes in the gut microenvironment and PD prevention. Long-term observational studies are also needed to determine the cumulative risk of PD in patients with IBD according to the KM treatment.

\section{Conclusions}

After adjusting for confounding variables, the results of this study showed that IBD patients in South Korea who received conventional and KM treatments had a significantly lower risk of PD compared to the risk in patients administered only conventional treatment, especially in those with mild CCI. Therefore, KM treatment may prevent PD in IBD patients. IBD patients should receive the $\mathrm{KM}$ treatment from the mild state of disease to prevent PD.

Supplementary Materials: The following are available online at http://www.mdpi.com/2077-0383/9/8/2422/s1, Table S1. Definitions of IBD drugs on the basis of main component codes and Table S2. Types of Korean medicine (KM) treatment. 
Author Contributions: Conceptualization, H.N., J.J., H.K. and S.-U.P.; Data curation, H.N., J.J., S.K., S.-Y.C., W.-S.J., S.-K.M., J.-M.P., C.-N.K., H.K. and S.-U.P.; Formal analysis, H.N., J.J., H.K. and S.-U.P.; Investigation, H.N. and J.J.; Methodology, H.N., J.J., H.K. and S.-U.P.; Writing-Original draft, H.N.; Writing-Review \& editing, S.K., S.-Y.C., W.-S.J., S.-K.M., J.-M.P., C.-N.K., H.K. and S.-U.P. All authors have read and agreed to the published version of the manuscript.

Funding: Basic Science Research Program through the National Research Foundation of Korea (NRF) funded by the Ministry of Education: NRF-2017R1D1A1B03031688.

Conflicts of Interest: The authors declare no conflict of interest.

\section{References}

1. Wang, Q.; Liu, Y.; Zhou, J. Neuroinflammation in Parkinson's disease and its potential as therapeutic target. Transl. Neurodegener. 2015, 4, 19. [CrossRef] [PubMed]

2. Ascherio, A.; Schwarzschild, M.A. The epidemiology of Parkinson's disease: Risk factors and prevention. Lancet Neurol. 2016, 15, 1257-1272. [CrossRef]

3. Sheehan, D.; Moran, C.; Shanahan, F. The microbiota in inflammatory bowel disease. J. Gastroenterol. 2015, 50, 495-507. [CrossRef]

4. Houser, M.C.; Tansey, M.G. The gut-brain axis: Is intestinal inflammation a silent driver of Parkinson's disease pathogenesis? NPJ Parkinsons Dis. 2017, 3, 3. [CrossRef]

5. Cersosimo, M.G.; Benarroch, E.E. Pathological correlates of gastrointestinal dysfunction in Parkinson's disease. Neurobiol. Dis. 2012, 46, 559-564. [CrossRef] [PubMed]

6. Rolli-Derkinderen, M.; Leclair-Visonneau, L.; Bourreille, A.; Coron, E.; Neunlist, M.; Derkinderen, P. Is Parkinson's disease a chronic low-grade inflammatory bowel disease? J. Neurol. 2019, 267, $2207-2213$. [CrossRef] [PubMed]

7. Leal, M.C.; Casabona, J.C.; Puntel, M.; Pitossi, F.J. Interleukin-1 $\beta$ and tumor necrosis factor- $\alpha$ : Reliable targets for protective therapies in Parkinson's Disease? Front. Cell Neurosci. 2013, 7, 53. [CrossRef]

8. Lin, J.C.; Lin, C.S.; Hsu, C.W.; Lin, C.L.; Kao, C.H. Association Between Parkinson's Disease and Inflammatory Bowel Disease: A Nationwide Taiwanese Retrospective Cohort Study. Inflamm. Bowel Dis. 2016, 22, 1049-1055. [CrossRef]

9. Peter, I.; Dubinsky, M.; Bressman, S.; Park, A.; Lu, C.; Chen, N.; Wang, A. Anti-Tumor Necrosis Factor Therapy and Incidence of Parkinson Disease Among Patients with Inflammatory Bowel Disease. JAMA Neurol. 2018, 75, 939-946. [CrossRef]

10. Weimers, P.; Halfvarson, J.; Sachs, M.C.; Saunders-Pullman, R.; Ludvigsson, J.F.; Peter, I.; Burisch, J.; Olén, O. Inflammatory Bowel Disease and Parkinson's Disease: A Nationwide Swedish Cohort Study. Inflamm. Bowel Dis. 2019, 25, 111-123. [CrossRef]

11. Villumsen, M.; Aznar, S.; Pakkenberg, B.; Jess, T.; Brudek, T. Inflammatory bowel disease increases the risk of Parkinson's disease: A Danish nationwide cohort study 1977-2014. Gut 2019, 68, 18-24. [CrossRef] [PubMed]

12. Park, S.; Kim, J.; Chun, J.; Han, K.; Soh, H.; Kang, E.A.; Lee, H.J.; Im, J.P.; Kim, J.S. Patients with Inflammatory Bowel Disease Are at an Increased Risk of Parkinson's Disease: A South Korean Nationwide Population-Based Study. J. Clin. Med. 2019, 8, 1191. [CrossRef] [PubMed]

13. Lee, J.; Lee, J.S.; Park, S.H.; Shin, S.A.; Kim, K. Cohort Profile: The National Health Insurance Service-National Sample Cohort (NHIS-NSC), South Korea. Int. J. Epidemiol. 2017, 46, e15. [CrossRef] [PubMed]

14. Kim, Y.I.; Kim, Y.Y.; Yoon, J.L.; Won, C.W.; Ha, S.; Cho, K.D.; Park, B.R.; Bae, S.; Lee, E.J.; Park, S.Y.; et al. Cohort Profile: National health insurance service-senior (NHIS-senior) cohort in Korea. BMJ Open 2019, 9, e024344. [CrossRef]

15. Park, S.; Chun, J.; Han, K.D.; Soh, H.; Choi, K.; Kim, J.H.; Lee, J.; Lee, C.; Im, J.P.; Kim, J.S. Increased end-stage renal disease risk in patients with inflammatory bowel disease: A nationwide population-based study. World J. Gastroenterol. 2018, 24, 4798-4808. [CrossRef]

16. Kang, E.A.; Han, K.; Chun, J.; Soh, H.; Park, S.; Im, J.P.; Kim, J.S. Increased Risk of Diabetes in Inflammatory Bowel Disease Patients: A Nationwide Population-based Study in Korea. J. Clin. Med. 2019, 8, 343. [CrossRef]

17. Choi, K.; Chun, J.; Han, K.; Park, S.; Soh, H.; Kim, J.; Lee, J.; Lee, H.J.; Im, J.P.; Kim, J.S. Risk of Anxiety and Depression in Patients with Inflammatory Bowel Disease: A Nationwide, Population-Based Study. J. Clin. Med. 2019, 8, 654. [CrossRef] 
18. Lee, J.; Im, J.P.; Han, K.; Kim, J.; Lee, H.J.; Chun, J.; Kim, J.S. Changes in Direct Healthcare Costs Before and After the Diagnosis of Inflammatory Bowel Disease: A Nationwide Population-Based Study. Gut Liver 2020, 14, 89-99. [CrossRef]

19. Lee, S.R.; Choi, E.K.; Rhee, T.M.; Lee, H.J.; Lim, W.H.; Kang, S.H.; Han, K.D.; Cha, M.J.; Cho, Y.; Oh, I.Y.; et al. Evaluation of the association between diabetic retinopathy and the incidence of atrial fibrillation: A nationwide population-based study. Int. J. Cardiol. 2016, 223, 953-957. [CrossRef]

20. Ha, J.E.; Jang, E.J.; Im, S.G.; Sohn, H.S. Medication Use and Drug Expenditure in Inflammatory Bowel Disease: Based on Korean National Health Insurance Claims Data (2010-2014). Korean J. Clin Pharm. 2019, 29, 79-88. [CrossRef]

21. Jang, S.; Park, C.; Jang, S.; Yoon, H.K.; Shin, C.S.; Kim, D.Y.; Ha, Y.C.; Lee, S.S.; Choi, H.J.; Lee, Y.K.; et al. Medical Service Utilization with Osteoporosis. Endocrinol. Metab. 2010, 25, 326-339. [CrossRef]

22. Kuhn, W.; Müller, T.; Nastos, I.; Poehlau, D. The Neuroimmune Hypothesis in Parkinson's Disease. Rev. Neurosci. 1997, 8, 29-34. [CrossRef] [PubMed]

23. Blum-Degen, D.; Müller, T.; Kuhn, W.; Gerlach, M.; Przuntek, H.; Riederer, P. Interleukin-1 Beta and interleukin-6 Are Elevated in the Cerebrospinal Fluid of Alzheimer's and De Novo Parkinson's Disease Patients. Neurosci. Lett. 1995, 202, 17-20. [CrossRef]

24. Müller, T.; Blum-Degen, D.; Przuntek, H.; Kuhn, W. Interleukin-6 Levels in Cerebrospinal Fluid Inversely Correlate to Severity of Parkinson's Disease. Acta Neurol. Scand. 1998, 98, 142-144. [CrossRef]

25. Sampson, T.R.; Debelius, J.W.; Thron, T.; Janssen, S.; Shastri, G.G.; Ilhan, Z.E.; Challis, C.; Schretter, C.E.; Rocha, S.; Gradinaru, V.; et al. Gut Microbiota Regulate Motor Deficits and Neuroinflammation in a Model of Parkinson's Disease. Cell 2016, 167, 1469-1480. [CrossRef]

26. Waljee, A.K.; Wiitala, W.L.; Govani, S.; Stidham, R.; Saini, S.; Hou, J.; Feagins, L.A.; Khan, N.; Good, C.B.; Vijan, S.; et al. Corticosteroid Use and Complications in a US Inflammatory Bowel Disease Cohort. PLoS ONE 2016, 11, e0158017. [CrossRef]

27. Kim, E.S.; Song, G.A.; Cho, K.B.; Park, K.S.; Kim, K.O.; Jang, B.I.; Kim, E.Y.; Jeon, S.W.; Lee, H.S.; Yang, C.H.; et al. Significant risk and associated factors of active tuberculosis infection in Korean patients with inflammatory bowel disease using anti-TNF agents. World J. Gastroenterol. 2015, 21, 3308-3316. [CrossRef]

28. Bonovas, S.; Minozzi, S.; Lytras, T.; González-Lorenzo, M.; Pecoraro, V.; Colombo, S.; Polloni, I.; Moja, L.; Cinquini, M.; Marino, V.; et al. Risk of malignancies using anti-TNF agents in rheumatoid arthritis, psoriatic arthritis, and ankylosing spondylitis: A systematic review and meta-analysis. Expert Opin. Drug Saf. 2016, 15, 35-54. [CrossRef]

29. Ji, J.; Lu, Y.; Liu, H.; Feng, H.; Zhang, F.; Wu, L.; Cui, Y.; Wu, H. Acupuncture and moxibustion for inflammatory bowel diseases: A systematic review and meta-analysis of randomized controlled trials. Evid. Based Complement. Alternat. Med. 2013, 2013, 158352. [CrossRef]

30. Bao, C.; Wang, D.; Liu, P.; Shi, Y.; Jin, X.; Wu, L.; Zeng, X.; Zhang, J.; Liu, H.; Wu, H. Effect of Electro-Acupuncture and Moxibustion on Brain Connectivity in Patients with Crohn's Disease: A Resting-State fMRI Study. Front. Hum. Neurosci. 2017, 11, 559. [CrossRef]

31. Zhao, C.; Bao, C.; Li, J.; Zhu, Y.; Wang, S.; Yang, L.; Shi, Y.; Liu, H.; Dou, C.; Ding, G.; et al. Moxibustion and Acupuncture Ameliorate Crohn's Disease by Regulating the Balance between Th17 and Treg Cells in the Intestinal Mucosa. Evid. Based Complement. Alternat. Med. 2015, 2015, 938054. [CrossRef] [PubMed]

32. Shang, H.X.; Wang, A.Q.; Chun-Hui Bao, C.H.; Wu, H.G.; Chen, W.F.; Wu, L.Y.; Ji, R.; Zhao, J.M.; Shi, Y. Moxibustion Combined With Acupuncture Increases Tight Junction Protein Expression in Crohn's Disease Patients. World J. Gastroenterol. 2015, 21, 4986-4996. [CrossRef] [PubMed]

33. Langhorst, J.; Wulfert, H.; Lauche, R.; Klose, P.; Cramer, H.; Dobos, G.J.; Korzenik, J. Systematic review of complementary and alternative medicine treatments in inflammatory bowel diseases. J. Crohns Colitis. 2015, 9, 86-106. [CrossRef] [PubMed]

34. Lim, H.D.; Kim, M.H.; Lee, C.Y.; Namgung, U. Anti-Inflammatory Effects of Acupuncture Stimulation via the Vagus Nerve. PLoS ONE 2016, 11, e0151882. [CrossRef] [PubMed]

35. Bao, C.H.; Wang, C.Y.; Li, G.N.; Yan, Y.L.; Wang, D.; Jin, X.M.; Wu, L.Y.; Liu, H.R.; Wang, X.M.; Shi, Z.; et al. Effect of mild moxibustion on intestinal microbiota and NLRP6 inflammasome signaling in rats with post-inflammatory irritable bowel syndrome. World J. Gastroenterol. 2019, 25, 4696-4714. [CrossRef] [PubMed] 
36. Pierce, C.A.; Haut, E.R.; Kardooni, S.; Chang, D.C.; Efron, D.T.; Haider, A.; Pronovost, P.J.; Cornwell, E.E., 3rd. Surveillance bias and deep vein thrombosis in the national trauma data bank: The more we look, the more we find. J. Trauma. 2008, 64, 932-937. [CrossRef]

37. Haut, E.R.; Pronovost, P.J. Surveillance bias in outcomes reporting. JAMA 2011, 305, 2462-2463. [CrossRef]

(C) 2020 by the authors. Licensee MDPI, Basel, Switzerland. This article is an open access article distributed under the terms and conditions of the Creative Commons Attribution (CC BY) license (http://creativecommons.org/licenses/by/4.0/). 